\title{
Open evacuation of pus: a satisfactory surgical approach to the problem of brain abscess?
}

\author{
RS MAURICE-WILLIAMS \\ From, the Royal Free Hospital and School of Medicine, London, UK
}

SUMMARY The operative management of intracerebral abscesses remains controversial, with both primary radical excision and repeated aspiration having their advocates. This paper describes a surgical technique which combines the advantages while avoiding the disadvantages of the two surgical approaches. At open operation the abscess is widely incised, all pus removed from within the capsule and any daughter loculi under direct vision and the empty capsule irrigated with antibiotic solution before closure of the wound without drainage. Fifteen cases were treated by this method. There were no deaths, 13 patients made full neurological recoveries and two were left partially disabled. In only one case was a second operation necessary to remove pus which had re-formed after an adequate primary clearance. There were no cases of wound sepsis or of late recurrence of the abscess.

Long after the introduction of antibiotics, suppuration within the brain carries a grave prognosis. The mortality reported after operation for brain abscess is still as high as over $40 \%$, and the survivors may be left with disabling neurological deficits. ${ }^{1-7}$ The type of operation which should be used remains controversial. Some neurosurgeons advocate primary radical excision of the abscess, ${ }^{8-11}$ whilst others favour repeated needle aspirations through a burr hole, late excision being reserved for those cases which fail to resolve with aspiration alone. ${ }^{72-15}$

This paper describes a method of surgical treatment which has not previously been proposed as a definitive approach, and which may be termed "open evacuation of pus". Through a generous exposure, the abscess capsule is widely incised and all pus cleared away under direct vision. The inside of the capsule is cleaned out with an antibiotic solution, the capsule is left in situ, and the wound closed in the normal way without drainage. The results of 15 cases treated by this method are described. This procedure would appear to combine the advantages, whilst avoiding the disadvantages, of the alternative conventional operations. The author believes that

Address for reprint requests: Mr RS Maurice-Williams, Department of Neurosurgery, Royal Free Hospital, Pond St, London, NW3 2QG, UK.

Received 12 October 1982 and in revised form 12 February 1983. Accepted 4 March 1983. open evacuation is the safest and most satisfactory way of treating the majority of cases of brain abscess.

\section{Method of treatment}

\section{(1) Pre-operative preparation}

Intravenous antibiotics in high dosage were begun as soon as the diagnosis was suspected. For an adult the most usual initial regime was: benzyl penicillin, 4 mega-units 6-hourly, chloramphenicol $500 \mathrm{mg}$ 6-hourly, metronidazole $500 \mathrm{mg}$ 6-hourly. Patients with clinical features suggesting marked cerebral compression or with CT scans showing extensive brain oedema around the abscess were started on intravenous dexamethasone, at an initial dose of $4 \mathrm{mg}$ 6-hourly, reducing to nil over 4-7 days. Immediately prior to operation each patient was given intravenous $20 \%$ mannitol to a total dose of $1 / 2 \mathrm{gm} / \mathrm{kg}$.

\section{(2) Operative technique}

All operations were carried out under general anaesthesia with assisted ventilation. Supratentorial abscesses were approached through an osteoplastic flap, infratentorial abscesses by a wide suboccipital craniectomy with removal of the arch of the atlas. The dura was widely opened and after the abscess capsule had been located by a brain cannula, a preliminary aspiration of pus was carried out through the cannula followed by an injection of antibiotic solution (penicillin 20000 units, plus either gentamicin $4 \mathrm{mg}$ or streptomycin $50 \mathrm{mg}$ in normal saline) into the capsule. The extradural tissues were draped off and a linear cortical incision $2-4 \mathrm{~cm}$ was made over the most superficial aspect of the abscess. This incision was deepened to reach 
the capsule, which was then widely opened. Using brain retractors and a headlamp, all residual pus was then cleared from within the capsule under direct vision. Care was taken to clear pus from any subsidiary loculi which had been revealed by the pre-operative CT scan. The inside of the capsule was then washed out with antibiotic solution and each layer of the closure was irrigated with a similar antibiotic solution, polybactrin spray also being used when this was available. The dura was closed, the brain invari- ably being slack after removal of the pus and the wound was closed in layers of interrupted silk in the usual fashion. Osteoplastic flaps were replaced and no drainage was used. After operation, high dosage antibiotics were continued for between 14 and 30 days, intravenously for the first few days. All patients received prophylactic anticonvulsants, which were continued indefinitely. Resolution of the abscess capsule and of the surrounding brain oedema was checked with serial CT scans.
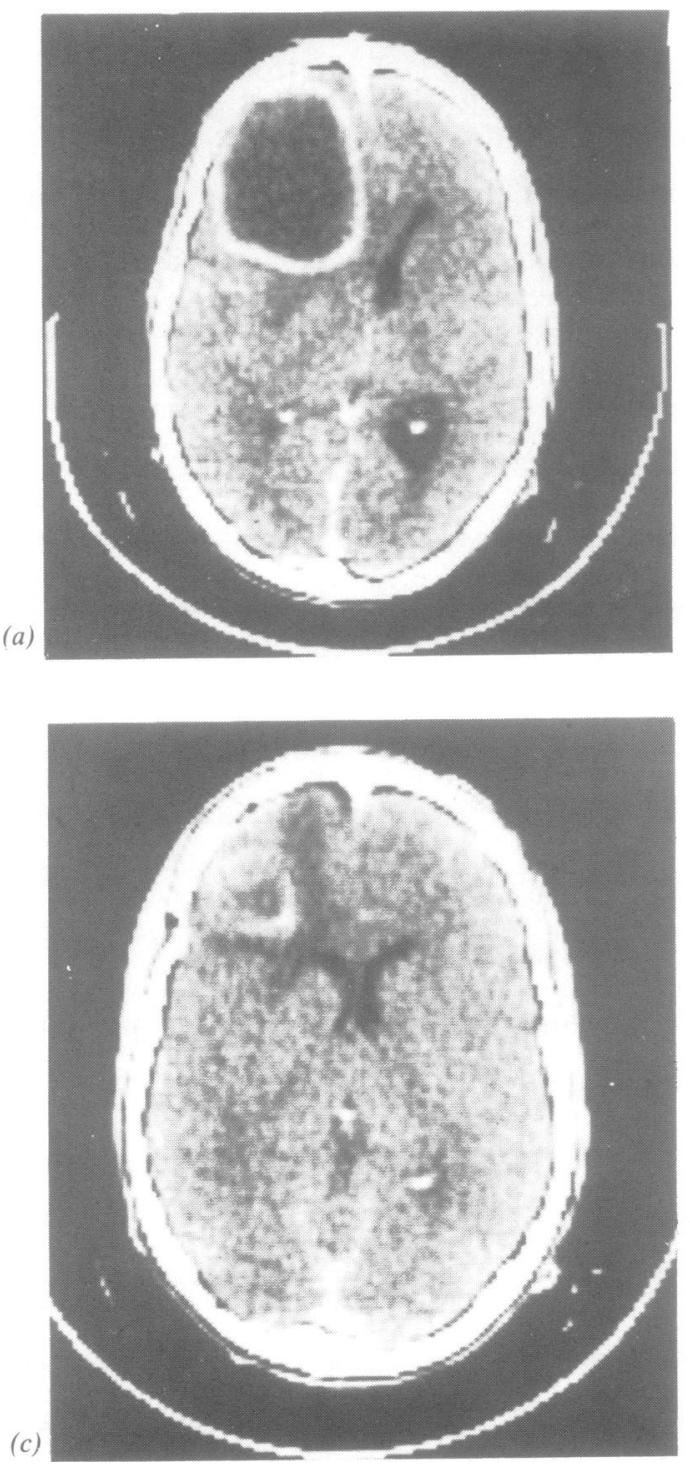
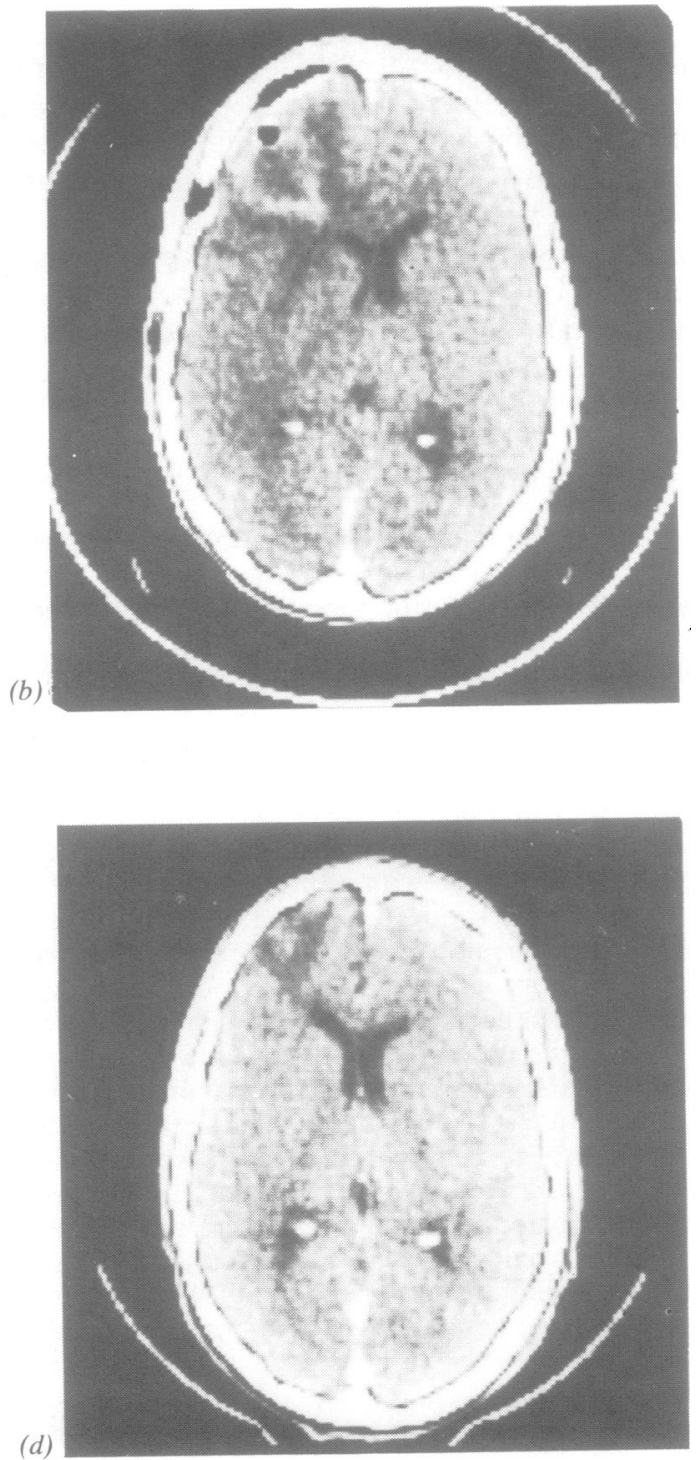

Fig 1 Serial CT scans (enhanced): large unilocular left frontal abscess secondary to frontal sinusitis. Open evacuation of pus via osteoplastic flap. (a) Prior to operation, (b) 6 days after operation, (c) 15 days after operation, (d) 15 months after operation. 

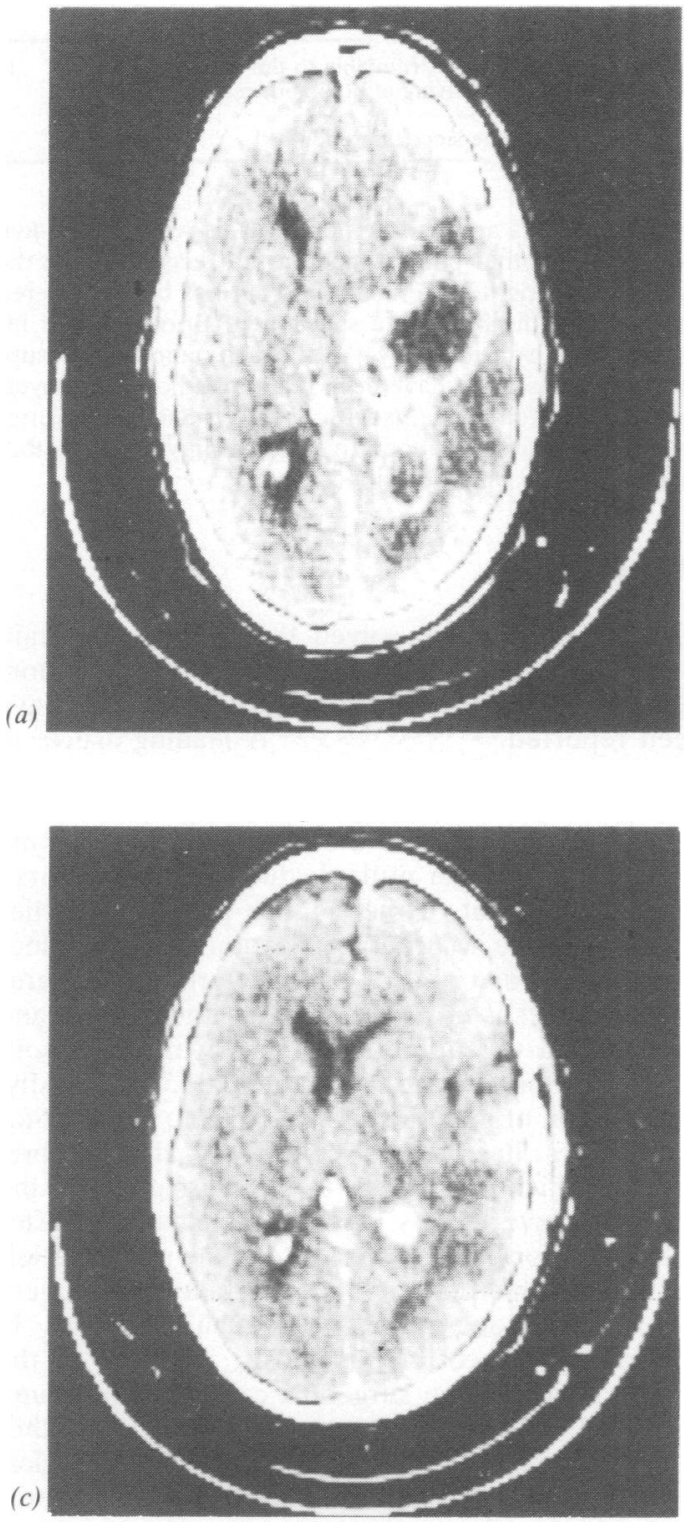

\section{Patients}

Over a five year period from 1977 to 1982,17 cases of intracerebral abscess were treated. Fifteen were treated by open evacuation, two by primary excision. Of the latter, one case was of a small superficial abscess in the right frontal premotor region. This patient made a full neurological recovery. In the other case, two separate abscesses were totally excised in the mistaken belief that they were infected hydatid cysts. This procedure inflicted a serious neurological deficit which might have been avoided had open evacuation been employed instead.

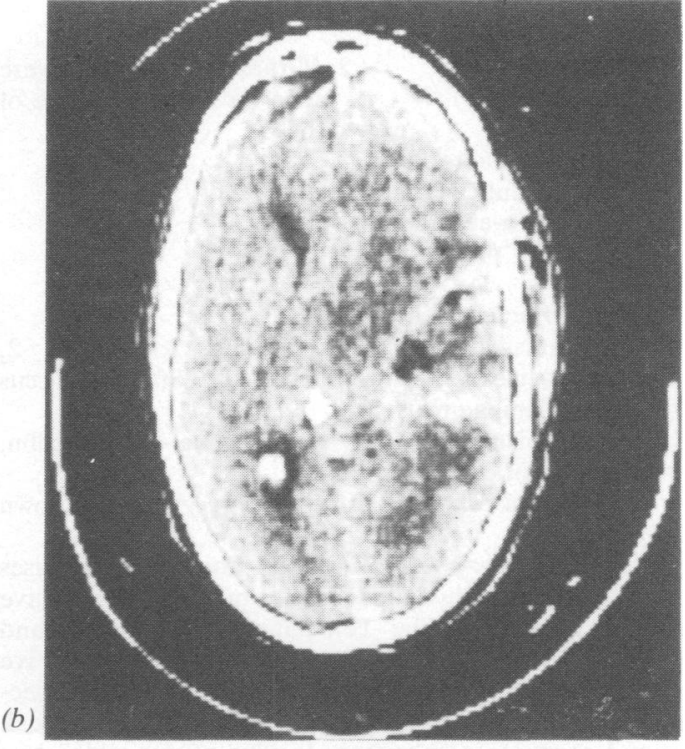

Fig 2 Serial CT scans (enhanced): multilocular right temporal otogenic abscess. Resolution of collapsed capsule and surrounding brain oedema. (a) Prior to operation, (b) 5 days after operation, (c) 17 days after operation.

Clinical details (of the 15 cases treated by open evacuation) (1) Site: The abscesses were situated as follows: parietooccipital 7 , frontal 3, temporal 3, cerebellar 2 . There were no cases of multiple abscess.

(2) Source of infection: Otogenic 4, secondary to frontal sinusitis 1 , blood-borne 10 (4 cases from dental sepsis, 2 from congenital cyanotic heart disease, source of infection uncertain in 4).

(3) Characteristics of the abscess: All were encapsulated and five had more than one loculus. One abscess had spontaneously burst into the subarachnoid space by the time of operation. In eight cases, the pus was noted to be thick- 
ened and difficult to aspirate through a brain cannula.

(4) Results of pus culture:

No organisms grown

2 (Gram positive cocci were seen on the film in one of these)

Anaerobic/micro-aerophilic

$$
\text { streptococci only } 6
$$

Anaerobic/micro-aerophilic

streptococci plus other

organisms 6

Proteus and Bacteroides 1

(Other organisms grown: bacteroides 2, proteus 2, fusiformis species 2, Enterobacter 1, Staphylococcus aureus 1, Melaningenicus 1)

All the organisms grown were sensitive to penicillin, chloramphenicol or metronidazole.

(5) Neurological condition prior to surgery: This is shown in Table 1

(6) Immediate post-operative course: There were no cases of acute or delayed wound sepsis, meningitis, infective cerebritis or septicaemia. Ten patients made rapid and uncomplicated recoveries after a single operation. Five patients underwent a second operation. In two cases a secondary deterioration proved to be due to a failure to break into and evacuate a subsidiary loculus of pus which had been visible on the original CT scan. Following reoperation and clearance of pus from these loculi, both patients recovered rapidly. Two more patients were subject to re-operation with negative findings at the abscess site when they failed to improve as rapidly as had been anticipated after the first operation, and when CT scans suggested persistence of the abscess. In both cases the abscess capsule was found to have collapsed and to contain a small slack quantity of sero-sanguinous fluid but no pus. In one of these cases the failure to improve seemed to be due to persistent cerebral oedema around the capsule. (This oedema soon cleared with the addition of dexamethasone to the antibiotic regime.) In the other case (a posterior fossa operation for cerebellar abscess) it may have been due to the formation of a CSF-filled cyst over the cerebellar surface. Only one patient required re-operation owing to re-formation of pus within a capsule which had been adequately cleared at the first operation: however, this patient was extremely debilitated and suffered from poorly controlled diabetes mellitus. The pus found at the second operation proved to be sterile.

(7) Eventual outcome at follow up from six months to four years: This is shown in table 2 . There were no deaths.

Table 1 Neurological condition of patients prior to operation

(a) Focal neurological deficit Present

(b) Signs of meningeal irritation Present Absent

(c) Conscious level Fully alert and orientated Drowsy, confused or irritable

Very drowsy, rousable only on vigorous stimulation Deeply unconscious
Table 2 Outcome at follow-up (6 months to 4 years)

No neurological deficit attributable to the abscess

Residual hemiparesis, living at home but requiring some support

Epilepsy (at any time since operation)

Twelve patients are leading normal lives with no residual disability. Another patient made a full recovery from the effects of his right parietal abscess but has been rendered permanently disabled by a subsequent thrombosis of his left common carotid artery. All thirteen patients with supratentorial abscesses have been maintained on anticonvulsants. Of the four patients with epilepsy, one had seizures in the immediate post-operative period only and the other three have had only occasional fits.

\section{Discussion}

Antibiotics have not solved the problem of brain abscess. Of recent years, series with operative mortalities ranging from under $10 \%$ to over $60 \%$ have been reported. ${ }^{12-4691011131516}$ A leading article in the British Medical Journal in 1977 estimated the overall surgical mortality of brain abscess to be about $40 \% .^{5}$ Since then there may have been some improvement in the outlook due to three factors. ${ }^{3}$ त The development of the CT has permitted earlier $\frac{\rho}{\mathrm{O}} \mathrm{O}$ diagnosis, more accurate localisation and the delineation of subsidiary loculi. The bacteriology of cerebral abscesses is now more clearly understood, and this has led to antibiotic regimes which are both more appropriate, and more adequate. ${ }^{71-20}$ Lastly,, 0 the addition of high doses of steroids to the antibio-? tic regimes has helped control of the cerebral oedema which contributes to the mass effect of the abscess. ${ }^{3}$ Nevertheless many patients still die after operations for brain abscess and many more are left severely disabled. Sometimes the failure of surgery is due to pre-operative factors such as a delay in arriving at the correct diagnosis, resulting in the patient reaching the surgeon's care in a moribund state with irreversible brain damage. ${ }^{1210}$ Other patients have died from a variety of pathological events which may have become worse after operation. Chief amongst these are spreading cerebral oedema with brain-stem compression, ${ }^{14810}$ intraventricular rupture of the abscess, ${ }^{4912}$ and dissemination of infection. ${ }^{14}$

The surgical approaches that have been used to treat brain abscess have been extensively reviewed by Turney and King. ${ }^{21}$ Prior to the development of antibiotics a variety of methods of external drainage or of marsupialisation were widely used. ${ }^{22-24}$ These procedures shared certain characteristics. The abscess capsule was encouraged to migrate towards the brain surface during the period that open drainage 
was maintained. At the same time methods were used to seal off the subarachnoid and subdural spaces around the drained area. By the use of meticulous technique amazingly good results were sometimes achieved. Sir William MacEwen reported in 1893 a series of 19 cases treated by external drainage with only one death..$^{25}$

Since the introduction of antibiotics, neurosurgeons have avoided external drainage, preferring instead either primary excision of the abscess or repeated aspiration through a burr-hole, each aspiration of pus being followed by the instillation of antibiotics into the capsule. Each method has its advantages and disadvantages and neither has gained a general acceptance.

Primary excision was first reported by Sargant in $1928,{ }^{26}$ and since that time several surgeons have advocated it as the method of choice ${ }^{8-1121}$. Primary excision may succeed in eradicating the intracerebral sepsis at a single operation. It provides an immediate and adequate cerebral decompression and for this reason it may be associated with a lower mortality than repeated aspiration. ${ }^{281016}$ Its main disadvantage is that complete excision of the capsule, which often has tentacular extensions into the surrounding oedematous white matter, may involve extensive damage to cerebral tissue. ${ }^{12} 16$ For this reason, excision has been associated with a higher incidence of residual neurological deficits than has aspiration, ${ }^{41316}$ although this has been disputed. ${ }^{89} \mathrm{~A}$ further problem is that primary excision may be a very major operation, especially if the patient is in a poor and deteriorating condition. Considerable surgical experience and skill may be required if the cerebral damage is to be minimised and if rupture into the ventricle of the deepest and thinnest part of the capsule is to be avoided. ${ }^{12}$

Aspiration involves a much smaller initial operation. It can be carried out under local anaesthesia if the patient is in a poor condition. However, it may do little to lessen the mass effect of the abscess, ${ }^{810} 15$ especially if the pus is thick, as it was noted to be in eight of 15 cases in the present series. Even if the pus is watery, much may be left behind after aspiration. The author has observed that when an apparently thorough aspiration of pus is immediately followed by open evacuation under the same anaesthetic, the capsule still contained a considerable quantity of pus. Furthermore, up to $40 \%$ of brain abscesses are multilocular, ${ }^{27}$ and if this feature is present, clearance of pus may be impossible even with repeated aspirations.

At best, aspiration requires repeated procedures. These may involve some trauma to the adjacent brain, as the thickening and collapsing capsule becomes more difficult to penetrate and the cannula glances off into the surrounding white matter. Eventual failure of the abscess to resolve may require a late secondary excision with infliction of even more neurological damage. ${ }^{89}$ Lastly aspiration alone is associated with a risk of late recurrence of the abscess in up to $8 \%$ of cases. ${ }^{416}$

Open evacuation has not previously been described as a definitive method of treating brain abscesses, although there seem to have been occasional cases treated by this approach in the preantibiotic era. ${ }^{28}$

Curiously, several cases treated with success by a similar method were described by Sir William MacEwen $^{25}$ in 1893. MacEwen believed that this method could be safely used in the treatment of acute abscesses, although he thought that more chronic abscesses with thick capsules required some form of external drainage. He stressed how necessary it was to carry out a thorough removal of all necrotic tissue, as well as fluid pus, from within the capsule. By irrigating the inside of the evacuated capsule with antiseptic solution and by dusting the bone surface and superficial tissues with iodoform and boracic powder, he was able to carry out a primary closure without sepsis, even though he sometimes re-implanted the bone disc removed by trephining. MacEwen's use of this method was not adopted subsequently by other surgeons. That he was able to carry out this procedure without complications at such an early date is a testimony to his quite extraordinary surgical skill and to the very high standards of care which his patients received.

Open removal of pus only became practicable as a general approach once antibiotics were invented, but since that time either excision or aspiration have been employed. Open evacuation shares with primary excision the advantage of removing the sepsis at a single operation. In only one of 15 cases did pus reform after an adequate initial clearance. The patient concerned was a cachetic man with neglected and unstable diabetes mellitus. Even so, the recurrent pus was sterile. In two further cases reoperation was required because the first operation had failed to remove pus from daughter loculi.

Like excision but unlike aspiration, open evacuation removes most of the mass effect of the abscess at the first operation. The capsule remains, but once the pus has been thoroughly removed the surrounding oedema soon resolves. The capsule generally collapses in and fades away on the CT scan over a period of 4-8 weeks.

Open evacuation adds little to the neurological damage inflicted by the suppuration itself. A $3-4 \mathrm{~cm}$ incision through the overlying brain is sufficient to permit a thorough inspection of the inside of the capsule. If the abscess is situated in an eloquent reg- 
ion of the brain, a wide exposure will generally permit the cortical incision to be placed so as to minimise any neurological damage. It is a feature of patients with brain abscesses that they may recover from apparently insuperable cerebral insults. One patient in this series had total aphasia, a dense right hemiplegia and a right homonymous hemianopia prior to the open evacuation of his left parietal abscess. Nine months later he had returned to his post as a professor of philosophy without any detectable neurological deficit.

It might be thought that open evacuation of pus would lead to dissemination of sepsis, as some bacterial contamination of the wound is inevitable, however great the care which has been taken to avoid spillage of pus and to screen off the surrounding tissues. This did not occur. There was no instance of wound, meningeal or systemic sepsis after operation, nor was there any case of late recurrence of the abscess within the capsule which had been left in situ. Replacement of free osteoplastic flaps was possible without infection. This lack of sepsis is probably a reflection of the fact that the organisms contained in encapsulated brain abscesses are generally of low virulence and are often obligate anaerobes $^{518}$ They are easily overwhelmed by appropriate systemic and topical antibiotics once necrotic material (pus) has been removed.

Appropriate antibiotics in high dosage by the intravenous route are an essential adjunct to the surgical removal of pus from the brain. Culture of the pus confirmed the findings of Garfield ${ }^{7}$ that anaerobic streptococci are the commonest organisms present. A combined regime of penicillin, chloramphenicol and metronidazole should be sufficient to combat any likely organisms, given in large intravenous dosage. All three antibiotics penetrate the capsule in effective concentrations. ${ }^{17}$ Penicillin will combat streptococci, while the gram negative bacteria which often accompany streptococci will be eradicated by either chloramphenicol or metronidazole..$^{51920}$ Irrigation of the inside of the capsule and the layers of the wound with penicillin and gentamicin prepared for intrathecal injection seems a wise additional precaution. Systemic antibiotics were deliberately commenced prior to surgery, up to 24 hours in some cases, with the intention of saturating the tissues with antibiotics before opening the abscess capsule, even if this should result in the occasional case where culture of the pus was negative.

The incidence of epilepsy after open evacuation is unremarkable. After treatment of a cerebral abscess, up to $70 \%$ of patients will eventually have at least one fit if the follow-up is sufficiently prolonged. ${ }^{29}$
Whether the capsule is removed or left in situ seems to make no difference to the risk of having fits ${ }^{101629}$ although at one time a lower incidence of epilepsy was thought to occur after excision than after aspiration.

Open evacuation is not an approach which is free of problems. It necessitates a major intracranial operation and is probably not an appropriate procedure for an inexperienced or partly trained neurosurgeon. The surgeon must minimise damage to the overlying brain when he incises the abscess and yet be sufficiently bold to clear all the pus from the main abscess cavity and from any daughter loculi. Failure to do this in two cases in this series necessitated a second operation. It is not suitable for the occasional case of unencapsulated focal brain suppuration which reaches the surgeon before the pathologist, nor may it be suitable for some cases of small abscesses which are deeply situated or multiple. Successful treatment of these categories of abscess has been reported recently with antibiotics alone..$^{30}$

The surgeon should not be misled by the CT scan appearances after open evacuation. The capsule may persist as a ring shadow which is only partially collapsed for several weeks after operation. This should not be a reason for re-exploring the abscess if the patient's condition is improving and the degree of cerebral shift shown by the scan is diminishing. In

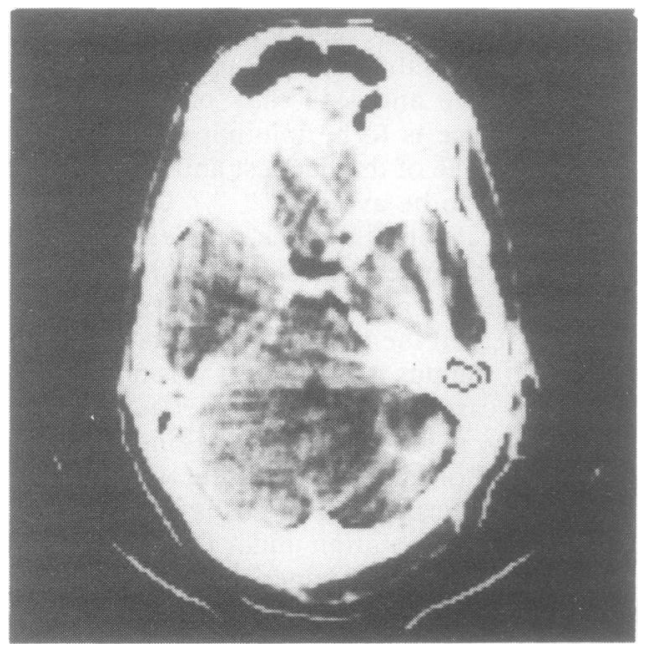

Fig 3 Misleading appearance of CT scan after open evacuation. CT scan with enhancement 5 weeks after open evacuation of otogenic abscess in right cerebellar hemisphere. Apparent persistence of abscess, but re-exploration revealed a collapsed capsule containing a small quantity of sterile sero-sanguinous fuid. Note lack of shift of fourth ventricle, indicating absence of mass effect. 
this series two cases were re-explored because of persistent scan abnormalities. In both the capsule contained nothing but a small amount of sterile sero-sanguinous fluid.

Having gained some experience of this approach, the author now believes that open evacuation is the surgical treatment of choice for most cases of brain abscess. A bold and thorough initial removal of pus combined with vigorous antibiotic treatment appears sufficient to effect a rapid recovery. The problems of repeated aspiration are avoided, while leaving the empty capsule within the brain does not lead to wound sepsis or to a late recurrence of the abscess.

Addendum: Since submission of this paper a further patient has been treated by this method. He was aged $28 \cdot \mathrm{yr}$ and had a unilocular left temporal abscess of unknown origin. Following open examination of pus he made a rapid and full recovery, without seizures. Two months later he has no neurological deficit and has returned to work.

\section{References}

${ }^{1}$ Carey, ME, Shelley NC, French LA. Experience with brain abscesses. J Neurosurg 1972;36:1-9

2 Yang S. Brain Abscess: a review of 400 cases. J Neurosurg 1981;55:794-9.

${ }^{3}$ Alderson D, Strong AJ, Ingham HR, Selkon JB. Fifteen-year review of the mortality of brain abscess. Neurosurgery 1981;8:1-6.

${ }^{4}$ Beller AJ, Sahar A, Praiss I. Brain abscess. Review of 89 cases over a period of 30 years. J Neurol Neurosurg Psychiatry 1973;36:757-8.

5 Treatment of cerebral abscesses-leading article. $\mathrm{Br}$ Med J 1977;2:978.

- McLelland CJ, Craig BF, Crockard HA. Brain abscesses in Northern Ireland: A 30 year community review. J Neurol Neurosurg Psychiatry 1979;41:1043-7.

${ }^{7}$ Garfield J. Management of supratentorial intracranial abscess: a review of 200 cases. Br Med J 1969;2:7-11.

${ }^{8}$ Choudhury AR, Taylor JC, Whitaker R. Primary excision of brain abscess. Br Med J 1977;2:1119-21.

' Ballantine HT, Shealey CN. The role of radical surgery in the treatment of abscess of the brain. Surg Gynaecol Obstet 1959;109:370-4.

${ }^{10}$ Kerr FWL, King RB, Meagher JN. Brain abscess-a study of forty-seven consecutive cases. JAMA 1958;168:868-72.
"Le Beau J, Creissard P, Harispe L, Redondo A. Surgical tretment of brain abscess and subdural empyema. J Neurosurg 1973;38:198-203.

${ }^{12}$ Northfield DWC. The Surgery of the Central Nervous System. Oxford, Blackwell. 1973.

${ }^{13}$ Lewin W. Recent developments in the management of brain abscess. $\mathrm{Br}$ Med J 1955;1:631-4.

14 Ohaegbulam SC, Saddequi NU. Experience with brain abscesses treated by simple aspiration. Surg Neurol 1980;13:289-91.

${ }^{15}$ Botterell EH, Drake CG. Localised encephalitis, brain abscess and subdural empyema. J Neurosurg 1952;9:348-66.

16 Jooma OV, Pennybacker JB, Tutton GK. Brain abscess; aspiration, drainage, or excision? J Neurol Neurosurg Psychiatry 1951;14:308-13.

${ }^{17}$ Black P. Graybill JR, Charache P. Penetration of brain abscess by systemically administered antibiotics. J Neurosurg 1973;38:705-9.

${ }^{18}$ Ingham HR, Seldon JB, Roxby CM. Bacteriological study of otogenic cerebral abscesses: chemotherapeutic role of metronidazole. $\mathrm{Br} \mathrm{Med} J$ 1977;2:991-3.

${ }^{19}$ De Louvois J, Gortvai P, Hurley R. Bacteriology of abscesses of the central nervous system: a multicentre prospective study. $\mathrm{Br}$ Med J 1977;2:981-4.

${ }^{20}$ De Louvois J, Gortvai P, Hurley R. Antibiotic treatment of abscesses of the central nervous system. $\mathrm{Br}$ Med J 1977;2:985-7.

${ }^{21}$ King JEJ, Turney F. Brain abscess: evolution of the methods of treatment. Ann Surg 1954;139:586-612.

${ }^{22}$ Horrax G. A method for the treatment of certain chronic encapsulated brain abscesses. Surg Clin NAm 1934;1179-86.

${ }^{23}$ King JEJ. The treatment of brain abscess by unroofing and temporary herniation of abscess cavity with the avoidance of usual drainage methods. Surg Gynecol Obstet 1924;39:554.

${ }^{24}$ Eagleton WP. Brain abscess: its surgical pathology and operative technic. New York, McMillan, 1922.

${ }^{25}$ MacEwen W. Pyogenic infective diseases of the brain and spinal cord. Glasgow, Maclehose, 1893.

${ }^{26}$ Sargent P. Remarks on drainage of brain abscess. $\mathrm{Br}$ Med J 1928;2:971.

${ }^{27}$ Stephanor S. Experience with multiloculated brain abscess. J Neurosurg 1978;49:199-203.

${ }^{28}$ Schreiber F. The treatment of cerebral abscesses by traction drainage. Am J Surg 1941;53:188.

${ }^{29}$ Legg NC, Gupta PC, Scott DF. Epilepsy following cerebral abscess. Brain 1973;96:259-68.

${ }^{30}$ Rosenblum ML, Hoff JT, Norman D, Edwards MS, Berg BC. Non-operative treatment of brain abscesses in selected high-risk patients. JNeurosurg 1980;52:217-25. 